\title{
An Improved Model for Predicting the Scattered S-N Curves
}

\author{
Jernej Klemenc1,* - Bojan Podgornik ${ }^{2}$ \\ 1University of Ljubljana, Faculty of Mechanical Engineering, Slovenia \\ 2Institute of Metals and Technology, Slovenia
}

\begin{abstract}
In this article an improved neural network model is presented that allows us to predict the scattered S-N curves. The model is capable of predicting the S-N curve in its high-cycle and very-high-cycle fatigue domains by considering also the increased scatter of the fatigue-life data below the knee point of the S-N curve. The scatter of the fatigue-life data for an arbitrary amplitude-stress level is modelled with a two-parametric Weibull's probability density function, the parameters of which are varied as a function of the amplitude-stress level. The parameters of the S-N curve trend and its scatter distribution are not fixed, but depend on the parameters of the production process via a serial-hybrid neural network. The article presents the theoretical background and the application in the case of real experimental fatigue data for 51CrV4 spring steel manufactured with two different manufacturing technologies and two different heat treatments.

Keywords: $\mathbf{5 1 C r V 4}$ steel, conventional manufacturing technology, electro-slag remelting, S-N curve, serial hybrid neural network

Highlights

- A model for predicting S-N curves and their scatter for 51 CrV4 steel was built.

- The model is capable of predicting the S-N curves in the transition zone between high-cycle and very high-cycle fatigue domains.

- $\quad$ The scatter of S-N curves is described by Weibull probability density function

- The model is based on a serial hybrid neural network.
\end{abstract}

\section{INTRODUCTION}

To evaluate structural reliability of dynamically loaded components it is necessary to know the scatter of the loading spectra as well as the scatter of the fatiguelife durability curve of the structural material [1] to [7]. Structures that are made from spring steels are typically subjected to a large number of load cycles. For this reason, a S-N fatigue-life curve is normally used to predict the fatigue life of such structures. In the past it was presumed that a fatigue-life limit $S_{a ; F L}$ exists at a certain number of load-cycles-to-failure $N_{F L}$, e.g., for structural steels the fatigue-life limit should occur between $2 \times 10^{6}$ [8] and $10^{8}$ [9] loadcycles-to-failure, see Fig. 1. However, it turned out that for most metallic structural materials there is no fatigue-life limit [10]. What exists in practice is a socalled Haibach's knee point, below which the slope of the S-N curve is reduced. This part of the S-N curve is usually referred to as a very-high-cycle domain. Haibach [11] proposed a model for the S-N curve in which the slope below its knee-point is $2 k-1$, if its slope in the high-cycle domain is $k$, see Fig. 1.

Additionally many other researchers have shown that the slope of the S-N curve in the very-high-cycle domain is reduced ([12] to [14]). What is common to all these cases is that, not only the slope, but also the scatter of the experimental fatigue-life data, is significantly changed (i.e. increased) below the knee point of the S-N curve, see Fig. 2.

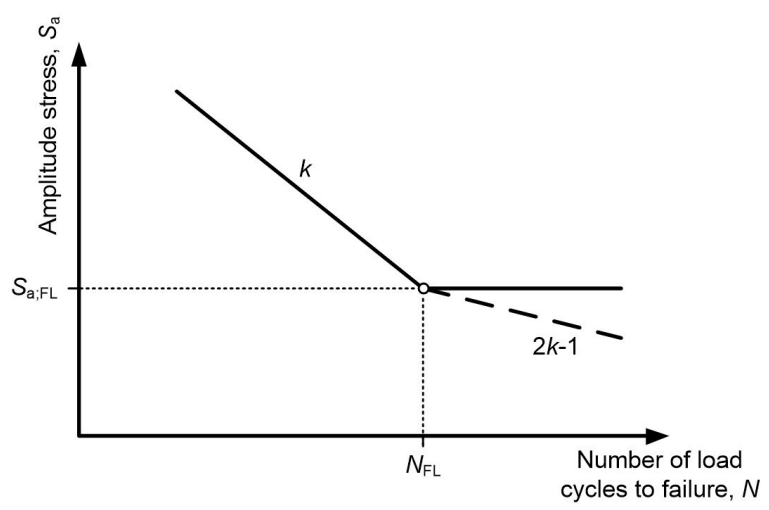

Fig. 1. A S-N fatigue-life curve

It was further shown [13] and [14] that the slope of the fatigue-life curve in the very-high-cycle domain is much smaller than the one proposed by Haibach. In order to make reliable predictions of the structure's fatigue life in the high-cycle and very-high-cycle domains one must be able to model the variable trend as well as the variable scatter of the number of loadcycles-to-failure for these two domains of the S-N curve.

The shape of the S-N curve in the vast neighbourhood of the knee point between the highcycle and very-high-cycle fatigue domains of the S-N 

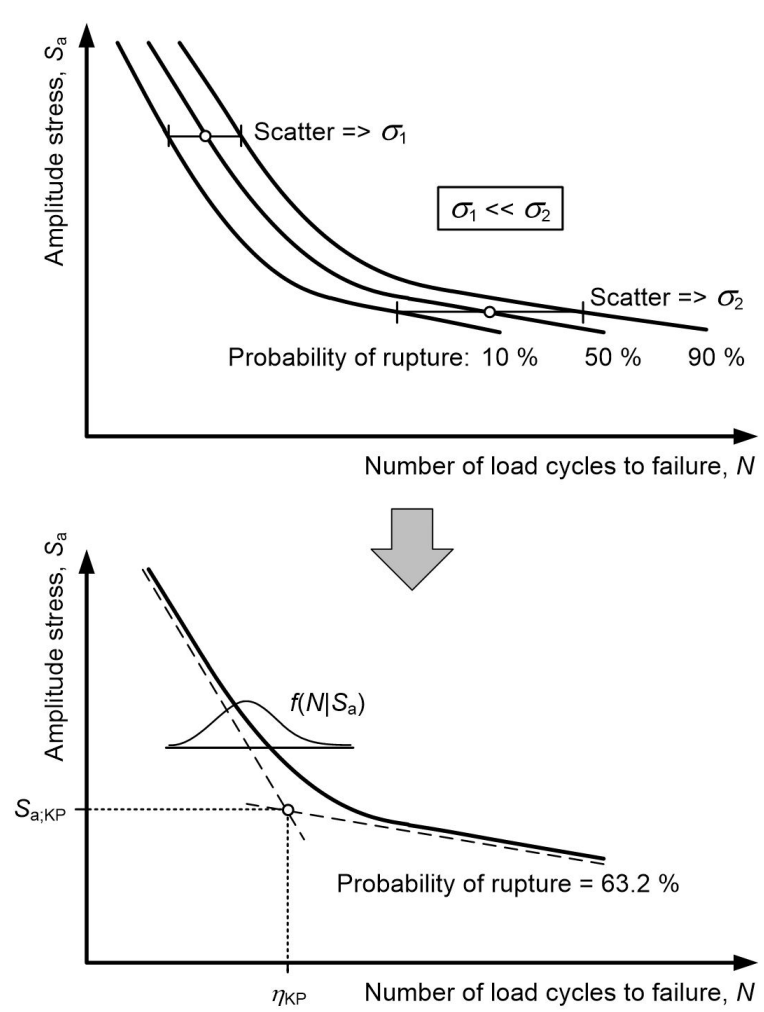

Fig. 2. Scatter of the S-N curve in the high-cycle and very-high-cycle fatigue domains

curve is similar to the shape of the Coffin-Manson curve [15] in the neighbourhood of the transition between the low-cycle elastic-plastic domain and the high-cycle elastic domain of the fatigue-life curve. For this reason it is possible to model the $\mathrm{S}-\mathrm{N}$ curve in the high-cycle and the very-high-cycle domains with a similar form:

$$
S_{a}=a \cdot N^{-b}+c \cdot N^{-(b+d)} ; \quad a, b, c, d>0,
$$

where $S_{a}$ represents the amplitude stress, $N$ is the number of load-cycles-to-failure and $a, b, c, d$ are parameters dependent on the material. We have shown before [16] that it is possible to model the fatiguelife curve of such a shape together with its scatter by applying a Weibull's two-parametric probability density function (PDF) to describe the scatter of the number of load-cycles-to-failure for an arbitrary amplitude-stress level:

$$
N \rightarrow f\left(N \mid S_{a}\right)=\frac{\beta}{\eta} \cdot\left(\frac{N}{\eta}\right)^{\beta-1} \cdot \exp \left[-\left(\frac{N}{\eta}\right)^{\beta}\right] .
$$

In that study we have presumed that the Weibull's scale parameter $\eta$ (which represents the number of load-cycles-to-failure at a 0.632 probability of rupture) was dependent on the amplitude-strain level $\varepsilon_{a}$ via Coffin-Manson equation and that the Weibull's shape parameter $\beta$ was constant [16]. However, there is a huge difference between the scatter of the $\varepsilon-\mathrm{N}$ curve and the scatter around the knee point between the high-cycle and very-high-cycle domains of the $\mathrm{S}-\mathrm{N}$ curve. While the width of the scatter band of the $\varepsilon-\mathrm{N}$ curve is constant below and above its knee point, this is not the case for the knee point between the highcycle and very-high-cycle domains of the S-N curve. In the latter case, the width of the scatter band of the $\mathrm{S}-\mathrm{N}$ curve in the very-high-cycle domain is much larger than in the high-cycle domain, see Fig. 2. For this reason it is not possible to describe the scatter of such durability curve using a two-parametric Weibull PDF from Eq. (2) with a constant shape parameter $\beta$. On the contrary, to model the S-N curve around the knee point between the high-cycle and very-highcycle fatigue domains the Weibull's shape parameter $\beta$ should also depend on the amplitude-stress level $S_{a}$.

The objective was to build a model for predicting the S-N curves and their scatter for a specific spring steel. Since the material's characteristics depend on the manufacturing technology and the heat treatment of such steels, these influential factors should be considered when modelling the corresponding S-N curves. The S-N curves can vary a great deal between differently produced and/or heat-treated steels, even for similar operating conditions. That is why we decided to apply a serial hybrid neural networks to build the model that is able to predict the S-N curve and its scatter as a function of the manufacturing technology and the heat treatment. A similar approach was successfully applied before, for modelling the dependence of the S-N and $\varepsilon-\mathrm{N}$ curves on the operating conditions ([17] and [18]). In the scientific literature there exist a number of articles on the application of neural networks for modelling and predicting durability curves. However, most of them are based on either multi-layer perceptrons or basis functions. With such an approach it is difficult to embed into a neural network an analytical model that is capable for predicting trend and scatter of the durability curve. Since the approach from Klemenc et al. [18] was proved to be successful we modified it in such a way that a new model is capable of predicting the S-N curve in its high-cycle and very-high-cycle fatigue domains by considering also the increased scatter of the fatigue-life data below the knee point of the S-N curve. The manufacturing technology and the heat treatment were the inputs to the neural network and the parameters of the S-N curve and its scatter were its outputs. 
Such a comprehensive model of the S-N curves with a capability of considering the increased scatter in the transition between the high-cycle and veryhigh-cycle domains has not been presented in the literature yet. A further innovation was to combine this model with the neural network in order to model the relationship between the parameters of the S-N curve and the applied manufacturing technology. The theory is explained in detail in section 1, experimental data are presented in section 2 and the results are presented and discussed in section 3 .

\section{THEORETICAL BACKGROUND}

\subsection{Variable-Scatter Model for the S-N Curve}

To model the S-N curves and their scatter for a $51 \mathrm{CrV} 4$ spring steel a similar statistical model as used in [16] was applied. Following this approach, the scatter of the number of load-cycles-to-failure $N$ for an arbitrary amplitude-stress level $S_{a}$ was described using a twoparametric Weibull's PDF, see Eq. (2) in section 1. The trend of the S-N curve in the neighbourhood of the knee-point was linked directly to the Weibull's scale parameter $\eta$ :

$$
S_{a}=a \cdot \eta\left(S_{a}\right)^{-b}+c \cdot \eta\left(S_{a}\right)^{-(b+d)} ; \quad a, b, c, d>0,
$$

with the Weibull's shape parameter $\beta$ that is now dependent on the loading level $S_{a}$ :

$$
\begin{aligned}
\beta=\beta\left(S_{a}\right)=f \cdot\left[1+\frac{g}{1+\exp \left[-h \cdot\left(S_{a}-S_{K P}\right)\right]}\right], \\
f \geq 1 ; \quad g, h, S_{K P}>0 .
\end{aligned}
$$

With this equation the shape parameter $\beta$ changes continuously and smoothly from the value of $f$ at very low amplitude-stress levels to the value of $(f \cdot g)$ at high amplitude-stress levels. The transition gradient between the two limit values depends on the parameter $h$, with the steepest gradient occurring at the amplitude-stress value $S_{K P}$. When combined with the scale parameter $\eta$ from Eq. (3) a small scatter is obtained at high amplitude-stress levels and an increased scatter is obtained at the lower amplitudestress levels that correspond to the very-high-cycle fatigue domain.

To avoid illogical shape of the durability curves around the knee point $S_{a}=S_{K P}$ two limit conditions are defined:

1. The parameter $g$ should never exceed the following value of $g_{\text {lim }}$ :

$$
g \leq g_{\lim }=\max \left\{3, \frac{b+d}{5 \cdot b}-1\right\} .
$$

2. The parameter $h$ should never exceed the following value of $h_{\text {lim }}$ :

$$
h \leq h_{\mathrm{lim}}=\frac{2 \cdot \min \left\{4, \frac{b+d}{5 \cdot b}\right\}}{S_{K P} \cdot f \cdot g} .
$$

The two conditions in Eqs. (5) and (6) limit the transition gradient between the small and large values of the shape parameter $\beta$ relative to the trend-curve slope ratio $(b+d) / b$. The knee-point stress $S_{K P}$ is calculated as a cross-section of the two asymptotes of Eq. (3), see Fig. 2:

$$
\begin{gathered}
S_{K P}=a \cdot \eta_{K P}^{b}, \\
\eta_{K P}=\exp \left[\frac{\ln (a)-\ln (c)}{-d}\right] .
\end{gathered}
$$

In this way the S-N curve and its scatter in the neighbourhood of the knee point between the high-cycle and very-high-cycle fatigue domains is modelled with seven parameters: $a, b, c, d, f, g$ and $h$. These parameters can be estimated using a numerical optimisation process.

\subsection{Serial Hybrid Multilayer Perceptron}

Since the material characteristics of the spring steel are dependent on the manufacturing technology and the heat treatment, they are also reflected in the trend and scatter of the corresponding S-N curve. This means that the parameters, $a, b, c, d, f, g$ and $h$ from subsection 1.1 are not constant, but they differ according to the applied production process. To model this relationship, the serial hybrid multilayer perceptron (SHMP) neural network according to Agarwal [19] was applied.

The multilayer perceptron (MP) is linked in series with the analytical model of the S-N curve and its scatter from Eqs. (3) and (4), see also Fig. 3 ([20] and [21]). This means that the MP is applied first for modelling the dependence of the parameters $a, b, c$, $d, f, g$ and $h$ on the manufacturing technology and the heat treatment. Then the analytical model is used only for modelling the S-N curve and its scatter after the seven parameters are predicted by the MP.

The result of each fatigue-life test was the number-of-cycles-to-failure $N_{l}$ for the given amplitude-stress level $S_{a ; l}$, the manufacturing technology and the heat treatment. Therefore the data points for training and/or testing the SHMP have the following form: $\left\{\left(\mathbf{x}_{l}, S_{a ; l}, N_{l}\right) l=1, \ldots, n\right\}$. The vector of the input independent variables $\mathbf{x}$ represents the 
parameters of the applied production process. The $l^{\text {th }}$ amplitude-stress level $S_{a ; l}$ is the independent variable of the analytical model from subsection 1.1 and Fig. 3. The $~_{\text {th }}$ number-of-cycles-to-failure $N_{l}$ is the SHMP's dependent variable and $n$ is the total number of sample points. Having this in mind, Eqs. (2) to (4) are changed as follows:

$$
\begin{gathered}
N=N\left(\mathbf{x}, S_{a}\right) \rightarrow f(N \mid \eta, \beta)=\frac{\beta}{\eta} \cdot\left(\frac{N}{\eta}\right)^{\beta-1} . \\
\cdot \exp \left[-\left(\frac{N}{\eta}\right)^{\beta}\right], \\
\eta=\eta\left(\mathbf{x}, S_{a}\right) \rightarrow S_{a}=a(\mathbf{x}) \cdot \eta\left(\mathbf{x}, S_{a}\right)^{-b(\mathbf{x})}+ \\
\beta=\beta\left(\mathbf{x}, S_{a}\right)=\quad+c(\mathbf{x}) \cdot \eta\left(\mathbf{x}, S_{a}\right)^{-(b(\mathbf{x})+d(\mathbf{x}))}, \\
=f(\mathbf{x}) \cdot\left[1+\frac{g(\mathbf{x})}{1+\exp \left[-h(\mathbf{x}) \cdot\left(S_{a}-S_{K P}(\mathbf{x})\right)\right]}\right] .
\end{gathered}
$$

The output of neuron $z_{j}$ is equal to the weighted sum of $M_{i}$ outputs $z_{i}$ from the preceding layer, modified by an activation function $\varphi_{j}$ :

$$
z_{j}=\varphi_{j}\left(\sum_{i=1}^{M_{i}} w_{i j} \cdot z_{i}+\Theta_{j}\right),
$$

where $w_{i j}$ are the synaptic weights and $\Theta_{j}$ is the threshold of the neuron. In our case the activation function was a $\tanh ()$ function for the hidden neurons and a linear function for the output neurons. This activation function was used instead of the more commonly used sigmoid function, because it turned out that a convergence of a training process was much better with the $\tanh ()$ than with the sigmoid function in the studied case. The inputs $x_{i}$ to the MP model were the components of the condition vector $\mathbf{x}$. The output neurons $z_{k}$ are applied for calculating the parameters $a, b, c, d, f, g$ and $h$. The relations between these parameters and the neural outputs $z_{k}$ must fulfill the restraints from Eqs. (3) and (4) and ensure the robustness of the model:

$$
\begin{gathered}
a=a(\mathbf{x})=10000 \cdot \frac{\exp \left(z_{k}^{a}\right)}{1+\exp \left(z_{k}^{a}\right)}, \\
b=b(\mathbf{x})=0.01+0.99 \cdot \frac{\exp \left(z_{k}^{b}\right)}{1+\exp \left(z_{k}^{b}\right)}, \\
c=c(\mathbf{x})=10^{7} \cdot \frac{\exp \left(z_{k}^{c}\right)}{1+\exp \left(z_{k}^{c}\right)}, \\
d=d(\mathbf{x})=0.01+0.99 \cdot \frac{\exp \left(z_{k}^{d}\right)}{1+\exp \left(z_{k}^{d}\right)}, \\
f=f(\mathbf{x})=1+4 \cdot \frac{\exp \left(z_{k}^{f}\right)}{1+\exp \left(z_{k}^{f}\right)}, \\
g=g(\mathbf{x})=3 \cdot \frac{\exp \left(z_{k}^{g}\right)}{1+\exp \left(z_{k}^{g}\right)}, \\
h=h(\mathbf{x})=0.1 \cdot \frac{\exp \left(z_{k}^{h}\right)}{1+\exp \left(z_{k}^{h}\right)} .
\end{gathered}
$$

These restraints were extensively tested in a preliminary study involving two different spring steels, a cold-drawn structural steel and two complexphase steels.

The cost function for the SHMP model was a maximum-likelihood function $E_{M L}$ according to

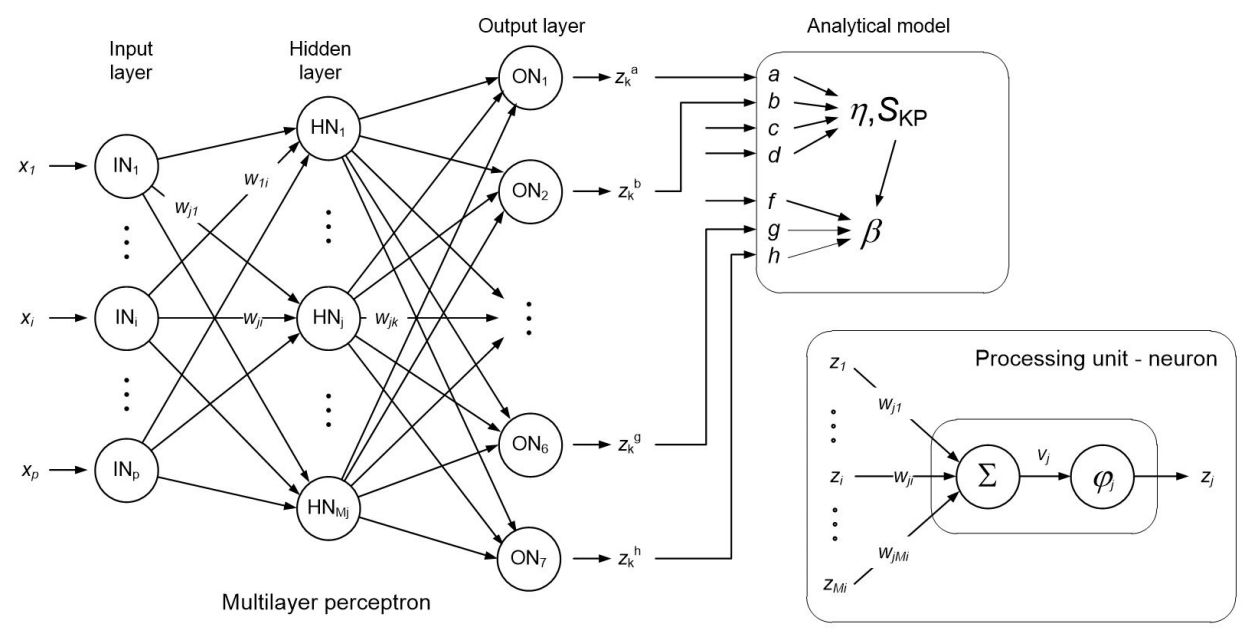

Fig. 3. Topology of the applied serial hybrid multilayer perceptron 


$$
\begin{aligned}
& E_{M L}=-\ln (M L)=-\ln \left(\prod_{l=1}^{n}\left[f\left(N_{l} \mid \eta_{\text {pred, }, l}, \beta_{\text {pred }, l}\right)\right]^{\delta_{l}} \cdot\left[1-F\left(N_{l} \mid \eta_{\text {pred }, l}, \beta_{\text {pred }, l}\right)\right]^{1-\delta_{l}}\right), \\
& E_{M L}=\sum_{l=1}^{n} e_{M L}(l)=\sum_{l=1}^{n}-\left\{\delta_{l} \cdot \ln \left[f\left(N_{l} \mid \eta_{\text {pred }, l}, \beta_{\text {pred }, l}\right)\right]+\left(1-\delta_{l}\right) \cdot \ln \left[1-F\left(N_{l} \mid \eta_{\text {pred }, l}, \beta_{\text {pred }, l}\right)\right]\right\}=
\end{aligned}
$$

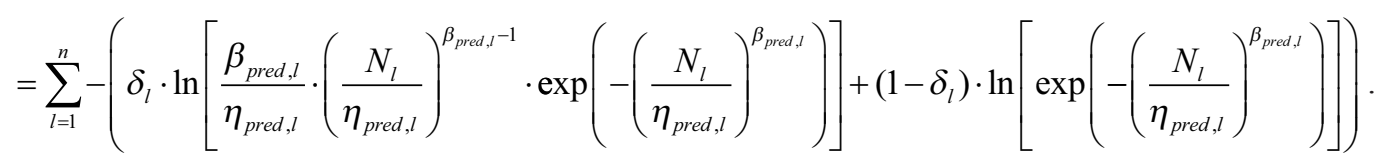

Pascual and Meeker [22]. Using this cost function the failed specimens as well as run-outs are considered for estimating the S-N curve and its scatter. For the fatigue failure a fatigue-failure identifier $\delta_{l}$ is set to 1 and for the run-outs it is set to 0 in the $E_{M L}$ cost function, see Eq. (13).

To calculate the error $e_{M L}(l)$ for each sample $l$, the parameters $a, b, c, d, f, g$ and $h$ are predicted first by the MP for the input vector $\mathbf{x}_{i}$. From these parameters and the value of $S_{a ; l}$ the Weibull's scale parameter $\eta_{\text {pred; }}$ is calculated with Eq. (9) using the Newton-Raphson method. The shape parameter $\beta_{\text {pred; } l}$ is calculated with Eq. (10).

The training of the SHMP model was carried out numerically with an error back-propagation algorithm and an epoch-based training. The gradients of the cost function $E_{M L}$ for the synaptic weights $w_{i j}$ were calculated as the sum of the gradients of the individual samples: $\partial E_{M L} / \partial w_{i j}=\sum_{l=1}^{n} \partial e_{M L}(l) / \partial w_{i j}$. In every iteration of the training process the synaptic weights were adapted as follows [23]:

$$
\Delta w_{i j}(t)=-\left.\theta_{i j}(t+1) \cdot \frac{\partial E_{M L}}{\partial w_{i j}}\right|_{(t)}+\alpha \cdot \Delta w_{i j}(t-1) .
$$

The training process proceeds until the modelled S-N curves with their scatter agree with the experimental results for the different vectors $\mathbf{x}_{i}$. The training-rate parameter $\theta_{i j}$ in Eq. (15) was added to consider the past gradient changes according to the delta-bar-delta rule [21].

The sample gradients $\partial e_{M L}(l) / \partial w_{i j}$ were calculated as follows ([21] and [22]):

$$
\partial e_{M L}(l) / \partial w_{i j}=\Delta_{j} \cdot z_{i},
$$

where $\Delta_{j}$ is the error of the neuron $j$ in the next layer to which the neuron $i$ sends its signal. The next layer of neurons is either a hidden or an output layer. To calculate the errors $\Delta_{j}$ in the output layer of the MP the partial derivatives $\partial e_{M L}(l) / \partial z_{k}$ for the output neurons $z_{k}$ must be calculated for each sample $\left(\mathbf{x}_{l}, S_{a ; l}, N_{l}, \delta_{l}\right)$ :

$$
\begin{aligned}
\frac{\partial e_{M L}(l)}{\partial z_{k}^{\text {par }}}= & \left\{\frac{\partial e_{M L}(l)}{\partial \eta_{\text {pred }, l}} \cdot \frac{\partial \eta_{\text {pred }, l}}{\partial p a r}+\frac{\partial e_{M L}(l)}{\partial \beta_{\text {pred }, l}} \cdot \frac{\partial \beta_{\text {pred }, l}}{\partial S_{K P}} \cdot \frac{\partial S_{K P}}{\partial p a r}\right\} . \\
& \cdot \frac{\partial p a r}{\partial z_{k}^{a}} ; \quad p a r=a, b, c, d, \\
\frac{\partial e_{M L}(l)}{\partial z_{k}^{\text {par }}}= & \frac{\partial e_{M L}(l)}{\partial \beta_{\text {pred }, l}} \cdot \frac{\partial \beta_{\text {pred }, l}}{\partial p a r} \cdot \frac{\partial p a r}{\partial z_{k}^{h}} ; p a r=f, g, h .(16)
\end{aligned}
$$

For reasons of clarity, the marking of the dependence on the vector $\mathbf{x}$ is omitted in Eq. (16) and the corresponding partial derivatives are listed in the appendix.

\subsection{Selecting the Optimal SHMP Model}

The SHMP model should represent the experimental data $\left\{\left(\mathbf{x}_{l}, S_{a ; l}, N_{l}, \delta_{l}\right) ; l=1, \ldots, n\right\}$ in the best possible way, but if the SHMP topology is too complex a data over-fit can occur. So the complexity should sometimes be sacrificed to avoid the over-fitting of the SHMP model. In our study we decided to choose the optimal topology of the SHMP model using the Akaike information criterion (AIC) [23]:

$$
A I C=2 \cdot n_{w}+2 \cdot \ln \left(E_{M L}\right),
$$

where $n_{w}$ is the number of synaptic weights in the MP and $E_{M L}$ is the final value of the cost function. The smaller the value of the AIC criterion, the better the SHMP topology. In addition to the original form of the AIC criterion, we also applied its modification with a second-order correction for small samples [24]:

$$
A I C_{c, B A}=A I C+\frac{2 \cdot n_{w} \cdot\left(n_{w+1}\right)}{n-n_{w}-1} .
$$

\section{EXPERIMENTAL DATA}

Cylindrical specimens according to the ASTM E 60692 standard [25] were prepared by Institute of Metals 
and Technology. They were cut from 51CrV4 steel bars in the rolling direction and manufactured by turning. In the middle the specimens were polished to an average roughness $R_{a}$ of $0.2 \mu \mathrm{m}$, see Fig. 4. The steel bars were produced by two manufacturing routes. The reference series was prepared by a conventional manufacturing technology, i.e. continuous casting and hot rolling. The alternative series was first continuously cast, then electro-slag remelted (ESR) in order to obtain more uniform microstructure and finally hot rolled.

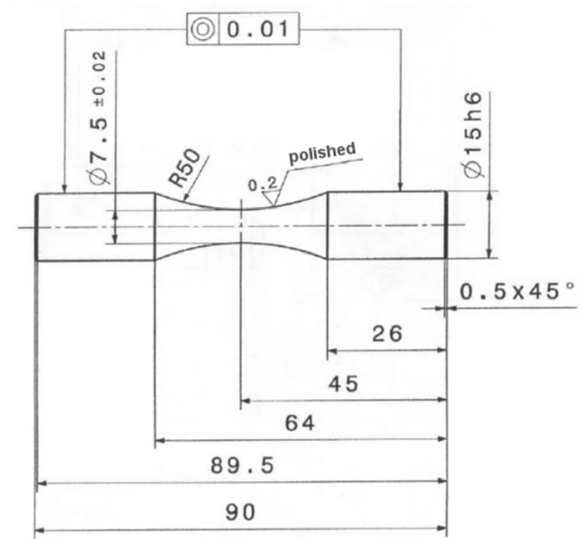

Fig. 4. Cylindrical specimen used for the fatigue experiments

Cylindrical test specimens from both series were subjected to two different heat treatment procedures. The first heat treatment of the specimens consisted of heating to $870{ }^{\circ} \mathrm{C}$, soaking for 10 minutes, which was followed by quenching in $N_{2}$ gas at 5 bar and a fast cooling rate of $7.5^{\circ} \mathrm{C} / \mathrm{s}$ until $60^{\circ} \mathrm{C}$. The second heat treatment of the specimens was performed at the same austenitizing temperature of $870{ }^{\circ} \mathrm{C}$ but a slower cooling rate of $2.7^{\circ} \mathrm{C} / \mathrm{s}$ was obtained by reducing $\mathrm{N}_{2}$ gas pressure to 1.05 bar. In both cases the specimens were finally tempered at $475^{\circ} \mathrm{C}$ for $1 \mathrm{~h}$.

Therefore four different groups of specimens were obtained: two different manufacturing technology routes combined with two different heat treatments. Altogether 101 specimens were manufactured with at least 23 specimens in each group [26].

The fatigue-life experiments were carried out at the universal dynamic servo-hydraulic test stand Instron 8802. A Dynacell load sensor was used for force measurements and the experiments were load-controlled. The axial loading of the cylindrical specimens was fully reversed $(R=-1)$. The experiments were carried out at a room temperature of $21{ }^{\circ} \mathrm{C}$ at different amplitude-stress levels (500 $\mathrm{MPa}$ to $780 \mathrm{MPa}$ ) until the fatigue failure occurred. The testing frequency was $30 \mathrm{~Hz}$. If the number of loading cycles exceeded 1 million without the fatigue failure the experiment run was terminated in order to reduce the experimental time and cost, since it follows from the literature that the fatigue failure between 1 million and 2 millions of loading cycles is highly unlikely for these kind of high-strength steels [12]. The experimental results are presented in Fig. 5.

\section{RESULTS AND DISCUSSION}

\subsection{Defining the SHMP Topologies and Pre-Processing the Data}

There are two independent parameters that represent the input for the SHMP (a binary variable indicating

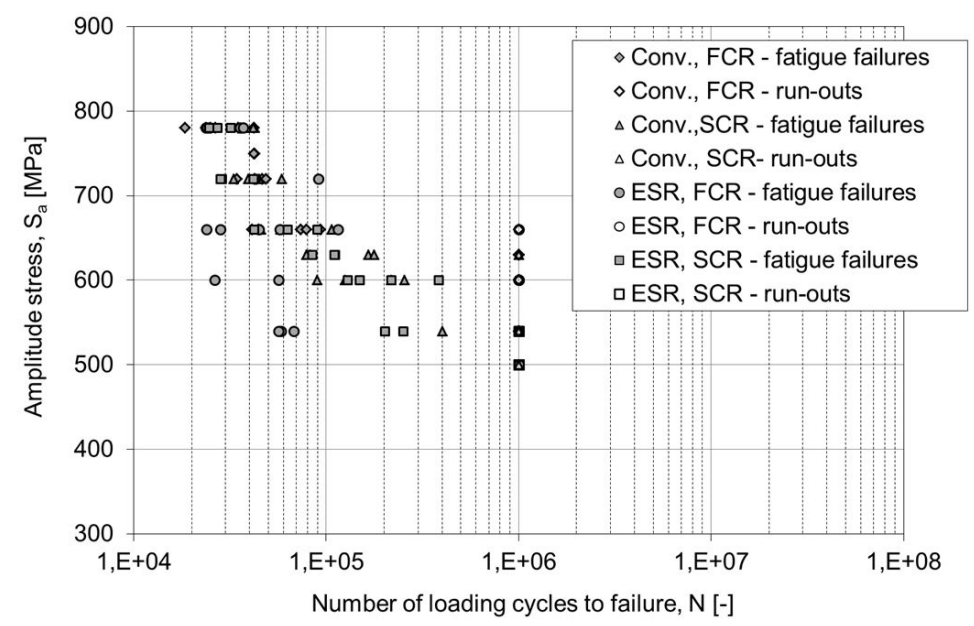

Fig. 5. Experimental fatigue-life data for $51 \mathrm{CrV} 4$ steel (abbreviations: conv. $=$ conventional manufacturing technology, $E S R=$ electro-slag remelting, $F C R=$ fast cooling rate, $S C R=$ slow cooling rate) 
the manufacturing technology and a real-valued variable indicating the cooling rate after the warmingup phase), so the number of neurons in the SHMP input layer was two. The seven parameters $a, b, c, d$, $f, g$ and $h$ were predicted by the SHMP. Therefore, there were seven neurons in the output layer of the SHMP. All the SHMPs had one hidden layer and four different topologies consisting of 2 (NN_1), 3 (NN_2), 6 (NN_3) or 9 (NN_4) neurons in the hidden layer.

The training set was composed of all the $n=$ 101 samples in Fig. 5. Many training processes were carried out with different initial values of the synaptic weights and training-rate parameters. A number of iterations were limited to 2,000,000 for each training process. The details on the training processes are listed in Table 1 for the best achieved cost functions. Variations of the cost-functions $E_{M L}$ during these training processes are presented in Fig. 6.

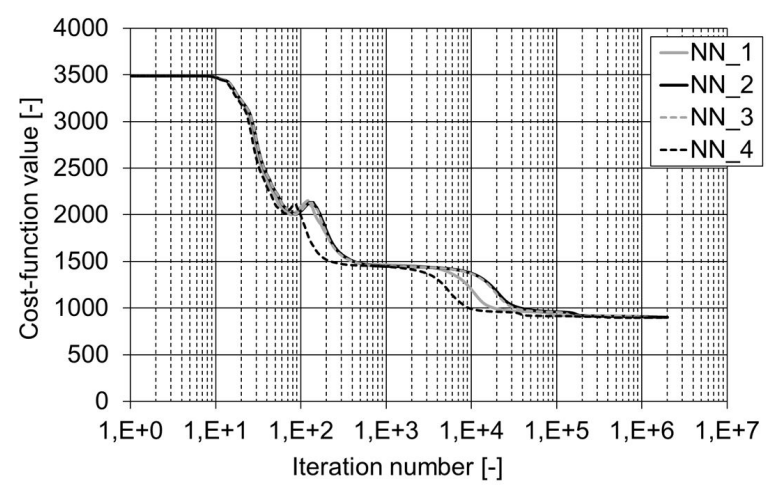

Fig. 6. A cost-function history during the training process

\subsection{Prediction Results}

We can see from Table 1 that the NN_4 topology with the largest number of neurons in the hidden layer resulted in the lowest value of the $E_{M L}$ cost function that was achieved before 1 million of training iterations with no further reduction in the costfunction, see Fig. 6.

However, this result is misleading, because the NN_4 topology had almost as much synaptic weights as there was the number of training samples. That is why this topology may be improper, since it is prone to data over-fitting.

From the viewpoint of the $A I C$ and $A I C_{c, B A}$ criteria, the best topology was $\mathrm{NN}_{-} 1$, with two neurons in the hidden layer. The good score in the two $A I C$ criteria was only due to its small number of synaptic weights, because its cost function was the highest. This implies that its fit of the S-N curves to the experimental data is not the best - see Figs. 7 and 8 for a comparison of the modelled S-N curves.

To assess the prediction quality of the SHMP models the S-N curves with their scatter were additionally estimated on a case-by-case basis. The seven parameters $a, b, c, d, f, g$ and $h$ for each of the four S-N curves were estimated using the real-valued genetic algorithm ([27] and [28]). The S-N curves, which were estimated using the genetic algorithm, are presented with a black color in Figs. 7 and 8.

It can be concluded from Table 1 and Figs. 7 and 8 that the best fit to the fatigue-life data is achieved with the NN_3 and NN_4 topologies. This

Table 1. Summary of the applied SHMP topologies and their training processes

\begin{tabular}{|c|c|c|c|c|c|}
\hline SHMP topology & & NN_1 & NN_2 & NN_3 & NN_4 \\
\hline \multirow{12}{*}{$\begin{array}{l}\text { Basic data about } \\
\text { the SHMP topologies } \\
\text { and their training } \\
\text { processes }\end{array}$} & No. of input neurons & 2 & 2 & 2 & 2 \\
\hline & No. of output neurons & 7 & 7 & 7 & 7 \\
\hline & Act. function of output neurons & linear & linear & linear & linear \\
\hline & No. of hidden layers and neurons & 1 layer, 2 neurons & 1 layer, 3 neurons & 1 layer, 6 neurons & 1 layer, 9 neurons \\
\hline & Act. function of hidden neurons & $\tanh ()$ & $\tanh ()$ & $\tanh ()$ & $\tanh ()$ \\
\hline & Initial value of the parameter $\eta$ & $10^{-8}$ & $10^{-8}$ & $10-8$ & $10^{-8}$ \\
\hline & Value of the parameter $\alpha$ & $10^{-2}$ & $10^{-2}$ & $10-2$ & $10^{-2}$ \\
\hline & Initial (final) value of the param. $\kappa$ & $5 \times 10^{-12}$ & $10^{-11}$ & $10-11$ & $5 \times 10^{-12}$ \\
\hline & Initial (final) value of the param. $\gamma$ & 0.995 & 0.99 & 0.99 & 0.995 \\
\hline & Value of the parameter $\xi$ & 0.7 & 0.7 & 0.7 & 0.7 \\
\hline & No. of train. Samples per epoch & 101 & 101 & 101 & 101 \\
\hline & Iteration no. for the minimum CF & 2000000 & 2000000 & 2000000 & 786200 \\
\hline \multirow{4}{*}{$\begin{array}{l}\text { Cost function values } \\
\text { and the AIC criteria } \\
\text { values for the trained } \\
\text { SHMP }\end{array}$} & Smallest value of the CF $E_{M L}$ & 901.53 & 901.39 & 897.41 & 896.71 \\
\hline & Number of synaptic weights $n_{w}$ & 27 & 37 & 67 & 97 \\
\hline & AIC criterion & 955.5311 & 975.3921 & 1031.407 & 1090.708 \\
\hline & $\mathrm{AlC}_{\mathrm{C}, \mathrm{BA}}$ criterion & 976.2434 & 1020.027 & 1307.528 & 7428.041 \\
\hline
\end{tabular}




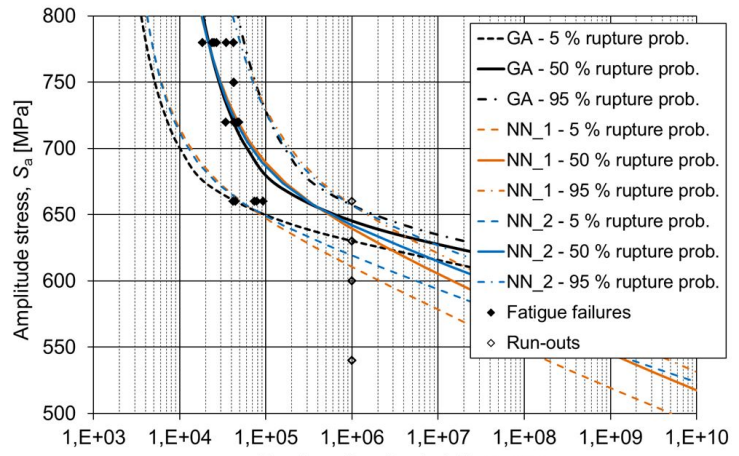

a) Number of cycles to failure, $N[-$

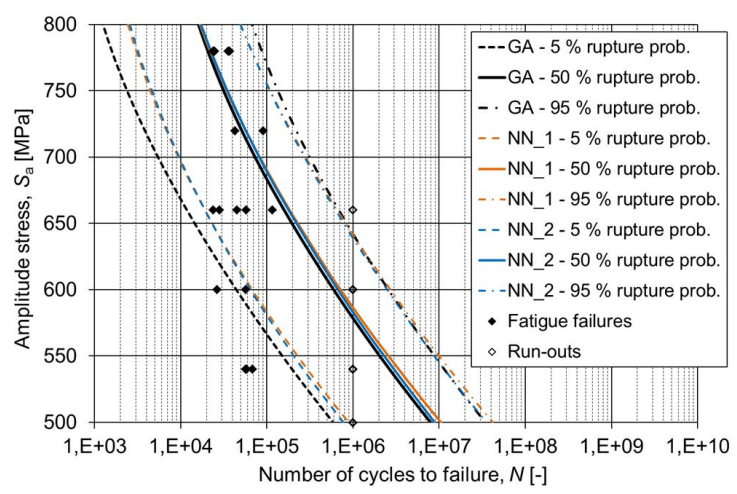

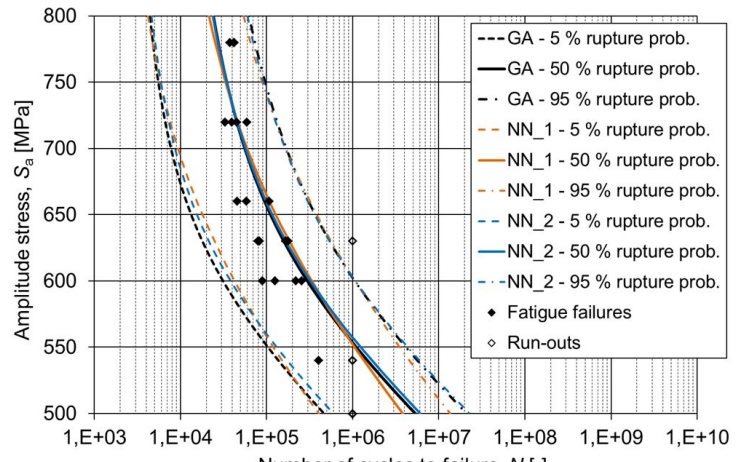

b)

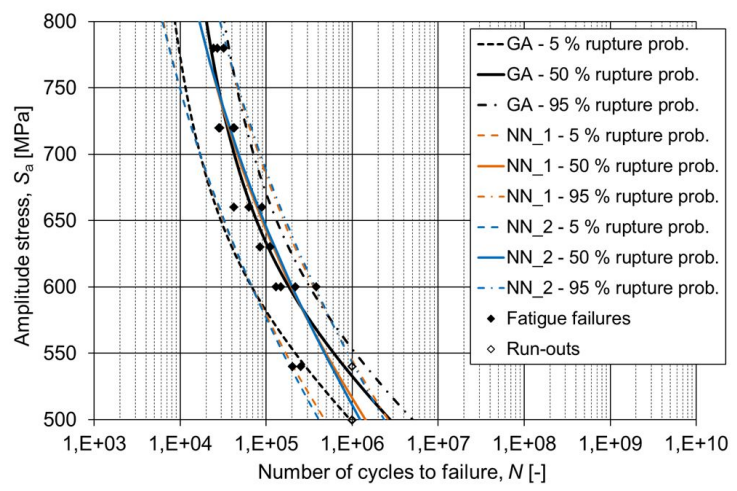

Fig. 7. Modelled S-N curves for the SHMP topologies NN_1 and NN_2; a) Conv., FCR, b) Conv., SCR, c) ESR, FCR, d) ESR, FCR

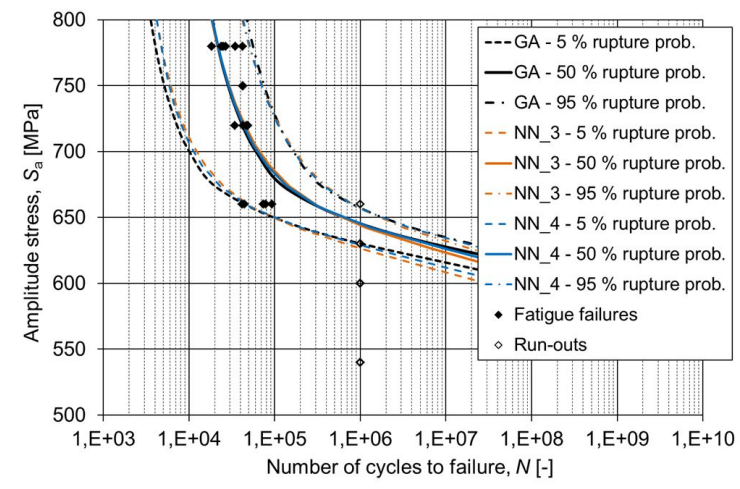

a)

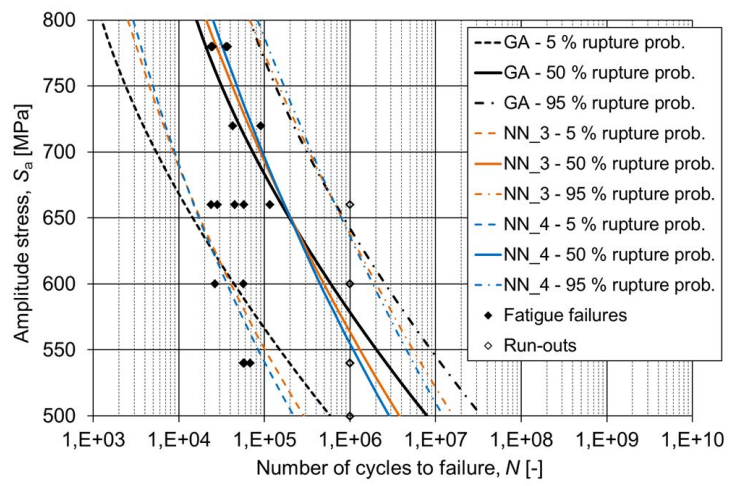

c)

Fig. 8. Modelled S-N curves for the SHMP topologies NN_3 and NN_4; a) Conv., FCR, b) Conv., SCR, c) ESR, FCR, d) ESR, SCR
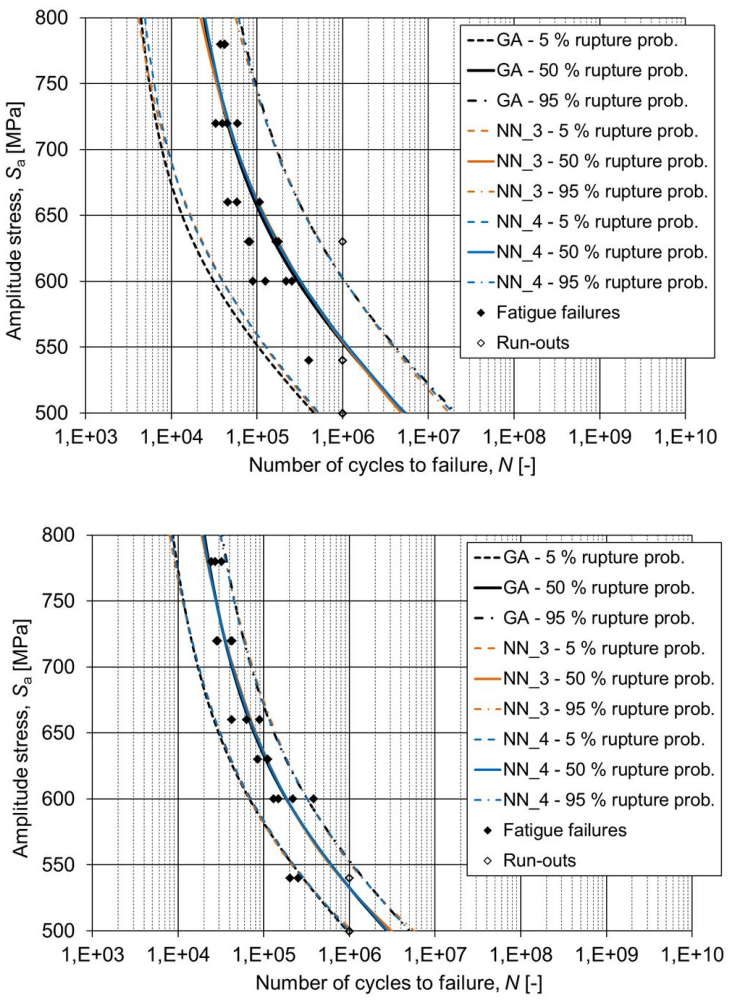

d) 
means that the most appropriate topology was NN_3 with less neurons in the hidden layer, because the discrepancy in the cost functions for the topologies is almost insignificant. Due to the relatively small number of training data the NN_3 topology has better generalisation ability and is less prone to the dataoverfitting. Besides, it resulted in the lower values of the two $A I C$ and criteria when compared to the NN_4 topology.

By comparing the S-N curves modelled with the SHMP and the genetic algorithm, it can be concluded that the modelled S-N curves do not differ a lot for the NN_3 and NN_4 topologies. This means that the SHMP topologies NN_3 and NN_4 were successfully applied for modelling the fatigue-life data of the $51 \mathrm{CrV} 4$ steel. The only exception is the S-N curve model for the data set that corresponds to the ESR manufacturing combined with the fast cooling rate during quenching. In this case the transition zone between the high-cycle- and very-high-cycle-fatigue domains is very broad in both directions $\left(S_{a}\right.$ and $\left.N\right)$. This is very difficult to model with a SHMP, given the fact that the same transition zone is much narrower for the other three data sets. For this reason it was extremely difficult to build a good S-N curve model, even if we try to do it individually with the genetic algorithm.

From the presented results it can be concluded that the introduced SHMP model is capable of modelling the S-N curve and its scatter well, also in the neighbourhood of a knee point between the high-cycle- and very-high-cycle-fatigue domains. Of course, since there is always a problem with a relatively small number of experimental fatiguelife data one should always try to find the simplest possible SHMP model that still enables fairly good predictions of the S-N curves and their scatter. The applied SMHP is general, which means that it can be applied for modelling the S-N curves and their scatter for arbitrary (metallic) materials, if the appropriate experimental data are available and the fatigue-life data-sets have approximately equal size for different manufacturing technologies.

\section{CONCLUSION}

In this article a procedure is presented for predicting the S-N curves and their scatter for $51 \mathrm{CrV} 4$ spring steel on the basis of experimental fatigue-life data that was obtained for different manufacturing technologies and heat treatments. The procedure is based on the application of a multilayer perceptron neural network, into which an analytical shape of the bi-linear S-N log-log curve was incorporated with the Weibull PDF describing its scatter. The results presented in the article were obtained for four combinations of the manufacturing technologies and quenching cooling rates. Different SHMP topologies were applied and each of them was trained with 101 experimentally obtained samples.

The results presented in the article show that it is possible to simultaneously estimate the parameters of the S-N curves and their scatter on the basis of the experimental data for different combinations of manufacturing technologies and heat treatments. The selection of a suitable SHMP topology should be made very carefully. It is almost always possible to obtain a good fit to the training data, if complex SHMP topologies with large numbers of neurons in the hidden layer are applied. On the other hand, such topologies can over-fit the data, especially if the number of synaptic weights in the SHMP model is comparable or larger than the number of data points in the training set. For this reason two variations of the AIC were applied in the research to select the proper SHMP topology. It turned out that the AIC criterion alone was not enough to estimate the most appropriate SHMP topology. So, when choosing the optimal SHMP model, one should make a trade-off between the simplicity of the SHMP model and its ability to generalise (but not over-fit) the experimental data. A general rule-of-thumb would be that there should be at least a few times more data points in the training set than there are synaptic weights in the SHMP model.

\section{ACKNOWLEDGEMENT}

The authors acknowledge the financial support from the Slovenian Research Agency (ARRS) for supporting the research programme P2-0182 R\&D evaluations.

\section{REFERENCES}

[1] Nagode, M., Klemenc, J., Fajdiga, M. (2001). Parametric modelling and scatter prediction of rainflow matrices. International Journal of Fatigue, vol. 23, no. 6, p. 525-532, DOI:10.1016/S0142-1123(01)00007-X.

[2] Tovo, R. (2001). On the fatigue reliability evaluation of structural components under service loading. International Journal of Fatigue, vol. 23, no. 7, p. 587-598, D0l:10.1016/ S0142-1123(01)00021-4.

[3] Zhao, Y.X., Yang, B., Zhai, Z.Y. (2008). The framework for a strain-based fatigue reliability analysis. International Journal of Fatigue, vol. 30, no. 3, p. 493-501, D0l:10.1016/j. ijfatigue.2007.04.006. 
[4] O'Connor, P., Kleyner, A. (2012). Practical Reliability Engineering, $5^{\text {th }}$ ed. John Willey \& Sons, New York.

[5] Xiao, Z., Zhao, Q.Y., Lin, F., Zhu, M.M., Deng, J.Y. (2018). Studying the fatigue life of a non-pneumatic wheel by using finite-life design for life prediction. Strojniški vestnik Journal of Mechanical Engineering, vol. 64, no. 1, p. 56-67, DOI:10.5545/sv-jme.2017.4695.

[6] Moral Portalés R., Bochons Sania M.d.M., Klemenc, J. (2018). Theoretical framework for estimating a product's reliability using a variable-amplitude loading spectrum and a stress-based approach. Fatigue and Fracture of Engineering Materials and Structures, vol. 41, no. 8, p. 1662-1673, DOl:10.1111/ffe.12804.

[7] Gróza, M., Váradi, K. (2018). Fatigue design of ferritic-pearlitic nodular cast iron components with surface discontinuities. Strojniški vestnik - Journal of Mechanical Engineering, vol. 64, no. 6, p. 373-382, D0l:10.5545/sv-jme.2017.5120.

[8] Buxbaum, 0. (1986). Betriebsfestigkeit. Verlag Stahleisen, Düsseldorf.

[9] EUROCODE 3 (2005). Design of Steel Structures - Part 1-9: Fatigue. European committee for standardization, Brussels.

[10] Bathias, C. (1999). There is no infinite fatigue life in metallic materials. Fatigue \& Fracture of Engineering Materials \& Structures, vol. 22, no. 7, p. 559-565, D0l:10.1046/j.14602695.1999.00183.x.

[11] Haibach, E. (2006). Betriebsfestigkeit, Verfahren und Daten zur Bauteilberechnung. 3. Auflage. Springer-Verlag, Berlin Heidelberg.

[12] Toplack, G., Eichlseder, W., Godor, I., Leitner, H. (2003). Influence of size and type of loading on S/N-Curve. ESIS Cumulative Fatigue Damage Conference Proceedings, p. 187191.

[13] Schaumann, P., Steppler, S. (2013). Fatigue tests of axially loaded butt welds up to very high cycles. Procedia Engineering, vol. 66, p. 88-97, D0l:10.1016/j.proeng.2013.12.065.

[14] Wagener, R., Hensel, J. (2014). Einflussgrößen auf die Lage des Abknickpunktes der Wöhlerlinie für den Schwingfestigkeitsnachweis von Schweiß verbindungen (IGF 16602N), from https://www.dvsev.de/fv/neu/indexcfm?Nav igation $=$ Forschungsergebnisse $\& P I D=783 \& C F I D=70346917$ \&CFTOKEN=97080438, accessed on 2018-26-10. Result score too low

[15] Dowling, N.E. (2012). Mechanical Behaviour of Materials, $4^{\text {th }}$ ed. Pearson Education, Upper Saddle River.
[16] Klemenc, J., Fajdiga, M. (2013). Joint estimation of E-N curves and their scatter using evolutionary algorithms. International Journal of Fatigue, vol. 56, p. 42-53, D0l:10.1016/j. ijfatigue.2013.08.005.

[17] Bučar, T., Nagode, M., Fajdiga, M. (2006). A neural network approach to describing the scatter of S-N curves. International Journal of Fatigue, vol. 28, no. 4, p. 311-323, D0l:10.1016/j. ijfatigue.2005.08.002.

[18] Klemenc, J., Janezic, M., Fajdiga, M. (2012). Modelling the scatter of E-N curves using a serial hybrid neural network. Neural Computing and Applications, vol. 21, no. 7, p. 15171530, DOI:10.1007/s00521-012-0828-2.

[19] Agarwal, M. (1997). Combining neural and conventional paradigms for modelling, prediction and control. International Journal of System Science, vol. 28, no. 1, p. 65-81.

[20] Bishop, C.M. (2005). Neural Networks for Pattern Recognition. Clarendon Press, Oxford.

[21] Haykin, S. (2009). Neural Networks and Learning Machines. Pearson education, New York.

[22] Pascual, F.G., Meeker, W.Q. (1999). Estimating fatigue curves with the random fatigue-limit model. Technometrics, vol. 41, no. 4, p. 277-290, DOI:10.2307/1271342.

[23] Akaike, H. (1974). A new look at the statistical model identification. IEEE Transactions on Automatic Control, vol. 19, no. 6, p. 716-723, D0I:10.1109/TAC.1974.1100705.

[24] Burnham, K.P., Anderson, D.R. (2002). Model Selection and Multimodel Inference: A Practical Information-Theoretic Approach, 2nd edition. Springer Verlag.

[25] ASTM E606-92(2004)e1: Standard Practice for StrainControlled Fatigue Testing. ASTM International, West Conshohocken, D0I:10.1520/E0606-92R04E01.

[26] Podgornik, B., Leskovšek, V., Godec, M., Senčič, B. (2014). Microstructure refinement and its effect on properties of spring steel. Materials Science \& Engineering: A, Structural Materials: Properties, Microstructure and Processing, vol. 599, p. 81-86, D0l:10.1016/J.msea.2014.01.054.

[27] Temby, L., Vamplew, P., Berry A. (2005). Accelerating real-valued genetic algorithms using mutation-withmomentum. The 18th Australian Joint Conference on Artificial Intelligence, vol 3809, Springer, Berlin, Heidelberg, DOI:10.1007/11589990_149e.

[28] Klemenc, J. (2015). Influence of fatigue-life data modelling on the estimated reliability of a structure subjected to a constant-amplitude loading. Reliability Engineering and System Safety, vol. 142, p. 238-247, DOI:10.1016/j.ress.2015.05.026.

\section{APPENDIX}

$$
\begin{aligned}
& \frac{\partial e_{M L}(l)}{\partial \eta_{\text {pred }, l}}=-\left\{\delta_{l} \cdot \frac{\beta_{\text {pred }, l}}{\eta_{\text {pred }, l}} \cdot\left[\left(\frac{N_{l}}{\eta_{\text {pred }, l}}\right)^{\beta_{\text {pred }, l}}-1\right]+\left(1-\delta_{l}\right) \cdot \frac{\beta_{\text {pred }, l}}{\eta_{\text {pred }, l}} \cdot\left(\frac{N_{l}}{\eta_{\text {pred }, l}}\right)^{\beta_{\text {pred }, l}}\right\},
\end{aligned}
$$

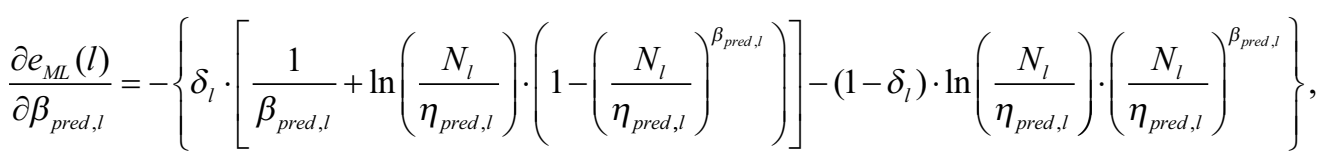




$$
\begin{aligned}
& \frac{\partial \beta_{\text {pred }, l}}{\partial S_{K P}}=-f \cdot g \cdot h \cdot \frac{\exp \left[h \cdot\left(S_{a, l}-S_{K P}\right)\right]}{\left\{1+\exp \left[h \cdot\left(S_{a, l}-S_{K P}\right)\right]\right\}^{2}}, \\
& \frac{\partial \eta_{\text {pred }, l}}{\partial a}=\frac{1}{a \cdot b \cdot \eta_{\text {pred }, l}^{-1}+c \cdot(b+d) \cdot \eta_{\text {pred }, l}^{-d-1}} \\
& \frac{\partial S_{K P}}{\partial a}=S_{K P} \cdot \frac{\partial \ln \left(S_{K P}\right)}{\partial a}=S_{K P} \cdot\left[\frac{1}{a} \cdot\left(1+\frac{b}{d}\right)\right], \\
& \frac{\partial a}{\partial z_{k}^{a}}=\frac{a}{1+\exp \left(z_{k}^{a}\right)} \\
& \frac{\partial \eta_{\text {pred }, l}}{\partial b}=\frac{-S_{a, l} \cdot \ln \left(\eta_{\text {pred,l }}\right)}{a \cdot b \cdot \eta_{\text {pred }, l}^{-b-1}+c \cdot(b+d) \cdot \eta_{\text {pred,l }}^{-b-d-1}}, \\
& \frac{\partial S_{K P}}{\partial b}=S_{K P} \cdot \frac{\partial \ln \left(S_{K P}\right)}{\partial b}=S_{K P} \cdot \frac{\ln (a)-\ln (c)}{d}, \\
& \frac{\partial b}{\partial z_{k}^{b}}=\frac{b-0.01}{1+\exp \left(z_{k}^{b}\right)} \\
& \frac{\partial \eta_{\text {pred }, l}}{\partial c}=\frac{1}{a \cdot b \cdot \eta_{\text {pred }, l}^{d-1}+c \cdot(b+d) \cdot \eta_{\text {pred }, l}^{-1}}, \\
& \frac{\partial S_{K P}}{\partial c}=S_{K P} \cdot \frac{\partial \ln \left(S_{K P}\right)}{\partial c}=S_{K P} \cdot\left[\frac{-b}{c \cdot d}\right], \\
& \frac{\partial c}{\partial z_{k}^{c}}=\frac{c}{1+\exp \left(z_{k}^{c}\right)} \\
& \frac{\partial \eta_{\text {pred }, l}}{\partial d}=\frac{-c \cdot \ln \left(\eta_{\text {pred }, l}\right)}{a \cdot b \cdot \eta_{p r e d, l}^{d-1}+c \cdot(b+d) \cdot \eta_{p r e d, l}^{-1}}, \\
& \frac{\partial S_{K P}}{\partial d}=S_{K P} \cdot \frac{\partial \ln \left(S_{K P}\right)}{\partial d}= \\
& =S_{K P} \cdot\left(\frac{-b}{d^{2}}\right) \cdot[\ln (a)-\ln (c)], \\
& \frac{\partial d}{\partial z_{k}^{d}}=\frac{d-0.01}{1+\exp \left(z_{k}^{d}\right)} \\
& \frac{\partial \beta_{\text {pred }, l}}{\partial f}=1+\frac{g}{1+\exp \left[-h \cdot\left(S_{a, l}-S_{K P}\right)\right]}, \\
& \frac{\partial f}{\partial z_{k}^{f}}=\frac{f-1}{1+\exp \left(z_{k}^{f}\right)}, \\
& \frac{\partial \beta_{\text {pred }, l}}{\partial g}=\frac{f}{1+\exp \left[-h \cdot\left(S_{a, l}-S_{K P}\right)\right]}, \\
& \frac{\partial g}{\partial z_{k}^{g}}=\frac{g}{1+\exp \left(z_{k}^{g}\right)} \\
& \frac{\partial \beta_{p r e d, l}}{\partial h}=f \cdot g \cdot\left(S_{a, l}-S_{K P}\right) \text {. } \\
& \cdot \frac{\exp \left[h \cdot\left(S_{a, l}-S_{K P}\right)\right]}{\left\{1+\exp \left[h \cdot\left(S_{a, l}-S_{K P}\right)\right]\right\}^{2}}, \\
& \frac{\partial h}{\partial z_{k}^{h}}=\frac{h}{1+\exp \left(z_{k}^{h}\right)},
\end{aligned}
$$

